\title{
Update on the current revolution in cancer immunotherapy
}

\author{
Ariana Nicole Renrick ${ }^{1,2,3}$, Zerick Terrell Dunbar ${ }^{1,2,3}$ \& Anil Shanker ${ }^{* 1,1,3,4,5}$ \\ ${ }^{1}$ Department of Biochemistry, Cancer Biology, Neuroscience \& Pharmacology, School of Medicine, Meharry Medical College, \\ Nashville, TN, USA \\ ${ }^{2}$ Department of Microbiology, Immunology \& Physiology, School of Medicine, Meharry Medical College, Nashville, TN, USA \\ ${ }^{3}$ School of Graduate Studies \& Research, Meharry Medical College, Nashville, TN, USA \\ ${ }^{4}$ Host-Tumor Interactions Research Program, Vanderbilt-Ingram Comprehensive Cancer Center, Vanderbilt University School of \\ Medicine, Nashville, TN, USA \\ ${ }^{5}$ Vanderbilt Institute for Infection, Immunology \& Inflammation, Vanderbilt University School of Medicine, Nashville, TN, USA \\ *Author for correspondence: Tel.: + 1615327 6460; Fax: +1 615327 6442; ashanker@mmc.edu
}

First draft submitted: 5 September 2018; Accepted for publication: 29 October 2018; Published online: 7 December 2018

Keywords: adoptive cell therapy • cancer immunology • CAR • cellular immunotherapy • checkpoint blockade • immunosurveillance $\bullet$ neoantigens $\bullet$ NK cells $\bullet$ T cells $\bullet$ tumor escape

In recent years, the field of cancer immunotherapy has seen outstanding progress due to our improved understanding of lymphocyte biology and its rapid translation into clinical trials. The field attracted this year's Nobel Prize in Medicine for the discovery of cancer therapy by inhibition of negative immune regulation. The journey of cancer immunology, however, has been long and arduous. The association between immune inflammation and tumors was observed by Rudolf Virchow as early as 1854. Building on this observation, Paul Ehrlich hypothesized that the immune system could surveil and inhibit tumor development [1]. Ehrlich stated: "In the enormously complicated course of fetal and postfetal development, aberrant cells become unusually common. Fortunately, in the majority of people they remain completely latent thanks to the organism's positive mechanisms" [1].

\section{Cancer immunosurveillance to immunoediting}

It was in the late 1950s that the concept of cancer immunosurveillance was formally introduced by Macfarlane Burnet and Lewis Thomas. The concept remained strongly debated until tumor immunosurveillance was unequivocally confirmed by immunologic rejection of tumors in experimental studies [2], and the high incidence of spontaneous and chemical-induced tumors observed in lymphocyte-specific gene-targeted immunodeficient mice [3]. The acceptance of the reality of cancer immunosurveillance led to rapid advancement in the field. Nonetheless, cancer heterogeneity and escape pose ongoing challenges hampering the outcomes of immunotherapy.

The tumor escape occurs often in all tumors under the pressure of endogenous immunosurveillance mechanisms against tumor, a process that was later described in the gamut of immunoediting [3]. The immunosurveillance hypothesis focused on the protection of host during the early stages of cellular transformation, whereas the immunoediting hypothesis ushered in the thought that the immune system protects the host as well as progressively edits tumor immunogenicity giving rise to three distinct phases of cancer elimination, equilibrium and escape [3]. The elimination phase embodies the original concept of immunosurveillance, where the innate and adaptive immune components work together to abolish transformed cells before they can establish within the host. Various mechanisms mediated by danger signals, such as type-I interferons, and stress ligands, such as those belonging to natural killer receptor group 2, member D (NKG2D) family of C-type lectin-like receptors, mediate a proinflammatory environment to recruit innate and adaptive immune cells to the site of tumor [3,4]. Studies have established the critical role for both the innate and adaptive immune effector cells that elicit tumor-specific responses to destroy potential tumors in the phase of cancer elimination [4,5].

The equilibrium phase allows atypical tumor cell variants to survive the elimination phase and remain in a state of dormancy maintained over a long period with immune ignorance [3]. The escape phase occurs as a result of Darwinian selection under the pressure of immune response, whereby tumor cells gain the ability to completely 
evade immune recognition and/or destruction facilitated by mechanisms including loss of cognate tumor antigen expression and an increased resistance to the cytotoxic effects of immune effector cells such as induction of antiapoptotic mechanisms [2,3]. Inflammatory cytokines, predominantly TNF- $\alpha$ and IFN- $\gamma$ present a feed-forward loop that helps sustain immunity against cancer as well as drive cancer progression [3]. Due to tumor escape and the resultant metastasis, survival rate in patients with metastatic cancers, who initially respond to immunotherapy, remains low in the range of 17 to $38 \%$.

\section{Early era of cancer immunotherapy to current checkpoint blockade therapy}

Induction of inflammation by direct tumor inoculation of bacterial products in 'Coley's toxins' by William Coley began the era of cancer immunotherapy more than 125 years ago at the turn of the twentieth century. However, those early approaches failed the clinical safety regulations. In late 1980s, the US FDA approved IL-2 as the first 'cancer breakthrough' treatment following the study showing positive effects in mice of the adoptive transfer of activated killer cells and IL-2 [6]. However, the therapeutic gains did not sustain due to multiple evasion mechanisms of tumors and immune compromise. Also, the natural immune checkpoint control of lymphocyte activation remained an issue when it came to sustaining antitumor responses. After vigorous work by multiple groups around the world, checkpoint blockade emerged as a second wave of 'cancer breakthrough' $\mathrm{T}$ cell immunotherapy with the discovery that cytotoxic T lymphocyte-associated protein 4 (CTLA-4) blocking antibodies could enhance antitumor immunity $[7,8]$. The CTLA- 4 is an intracellular protein that translocates to the cell surface to outcompete with CD28 for binding to costimulatory molecules like CD80 and CD86, thus generating an inhibitory signal characteristic of the contraction phase of $\mathrm{T}$ cell function [7].

Ipilimumab and tremelimumab, human CTLA-4-blocking antibodies, entered clinical trials in patients with advanced cancer about two decades ago. After two large-scale Phase III trials ipilimumab significantly increased survival in patients with metastatic melanoma [8]. These results were compared with patients who were treated with either tumor peptide vaccines or standard chemotherapy. With these findings, in 2011 the US FDA approved ipilimumab for melanoma treatment. Tremelimumab and other CTLA-4-blocking antibodies are still being tested in clinical trials [8]. Ipilimumab has been found to have issues with low or delayed response rates in patients. A profile of neoantigens conducted on tumor samples from patients with or without long-term clinical benefits to CTLA- 4 treatment revealed that patients with a higher tumor mutational landscape correlated with a strong antitumor response following CTLA- 4 blockade. A neoantigen signature was confirmed in a set of 39 patients with melanoma, who were then treated with ipilimumab and T cells activated by the predicted neoantigens [9].

Another pivotal immune checkpoint, characteristic of $\mathrm{T}$ cell exhaustion, is the programmed cell death receptor 1 (PD-1) molecule that negatively regulates antitumor $\mathrm{T}$ cell effector function following engagement with its ligand, programmed cell death ligand 1 (PD-L1), expressed in tumor microenvironment on the surface of tumor cells or other immune cells $[8,10]$. The PD- 1 blockade has been deemed more efficient compared with CTLA- 4 blockade because of its increased therapeutic activity and reduced toxicity [8]. Currently, the US FDA has approved five anti-PD-1 or anti-PD-L1 antibodies for the treatment of 11 different cancers. Pembrolizumab and nivolumab were the first PD-1-blocking antibodies approved by the US FDA for the treatment of refractory melanoma in 2014 and advanced non-small-cell lung cancer in 2015. Furthermore, atezolizumab was the first anti-PD-L1 antibody approved for urothelial cancers in 2016 and avelumab followed in 2017 for the treatment of Merkel cell carcinoma [8]. The PD-1-pathway blockade has shown elevated response rates in patients with melanoma, nonsmall-cell lung cancer, head and neck, gastroesophageal and bladder and urothelial cancers, as well as hepatocellular carcinoma caused by viral infections [8]. Due to the need to improve treatment response rates, combination CTLA- 4 and PD-1-blockade therapy is presently being explored. Clinical trials involving both ipilimumab and nivolumab have shown a significant increase in response rate as well as patient survival [8].

\section{Limitations of checkpoint therapy}

A caveat to combination checkpoint treatments is that with increased therapeutic benefit there is also increased toxicity, which surpasses either single-agent treatment alone [8]. Another foremost concern with the effectiveness of checkpoint blockade is the desensitization of $\mathrm{T}$ cells to checkpoint treatments. Immune resistance to checkpoint therapy can take place in a multitude of ways, one of which involves tumor cell evolution as a result of mutational burden that generates neoantigens, some of which lend immunogenicity but some can render $\mathrm{T}$ cells unresponsive to tumor cells [11]. Also, PD-L1 expression is linked to IFN- $\gamma$ receptor signaling, which is dependent on Janus family tyrosine kinases, JAK1 and JAK2. Consequently, a loss-of-function mutation in either JAK1 or JAK2 genes 
can render the use of PD-1 or PD-L1 blocking antibodies ineffective [12]. To overcome these mechanisms of resistance, combination therapy involving checkpoint blockade and other therapeutic agents are being investigated. For example, along with checkpoint therapies, the tumor microenvironment is being treated with IFN- $\gamma$-inducing Toll-like receptor agonists and oncolytic viruses. In addition, therapies such as chemotherapy, radiotherapy or oncogene-targeted therapies are used in conjunction with checkpoint blockade [8]. Although these approaches have proved to be effective in reducing tumor load, they also caused a host of toxicities related to tissue-specific inflammation, precluding long-lasting benefits in patients.

\section{Genetically engineered chimeric antigen receptors T cell therapy}

A recent promising therapy is being offered by genetic engineering of the $\mathrm{T}$ cell receptor on antitumor $\mathrm{T}$ cells. One of the latest in this modality is the engineering of chimeric antigen receptors (CAR), which are recombinant receptors consisting of an extracellular cognate antigen-binding domain, a hinge region, a transmembrane domain to anchor the CAR to the cell membrane and an intracellular signaling domain that mediate $\mathrm{T}$ cell activation. Three different types of structures can make up the target antigen-binding domain: single-chain variable fragment consisting of light and heavy chain portions ( $\mathrm{scFv}$ ) originating from antibodies, antigen-binding fragment (Fabs) chosen from libraries or natural ligands [13]. In contrast to the T cell receptor, which has to be matched to the patients' major histocompatibility (MHC) haplotype, CARs recognize epitopes without a need for peptide processing or MHC conjugation. Therefore, CARs are not limited by MHC restriction and can circumvent the traditional dynamics of lymphocyte activation. Moreover, CARs can recognize proteins, glycoproteins and glycolipids as potential targets. Following ligand engagement, CARs are designed to sustain full activation signals via the CD3 $\zeta$ chain and costimulation signals via a host of molecules such as CD28, CD27, CD134 (OX40), CD137 (4-1BB), DAP10 or ICOS. Incorporation of additional costimulatory molecules has led to the evolution of CAR T cells. First-generation CARs only involve activation with one costimulatory element, whereas second-generation contains an additional costimulatory molecule (dual signaling). Third-generation CARs have $\geq 3$ costimulatory molecules. For antitumor CAR T cell therapy to be clinically effective, several requirements should be met: sufficient numbers of patients' tumor-infiltrating $\mathrm{T}$ cells, which could prove challenging when a patient is immunocompromised from a first-line chemotherapy that affects $T$ cells; engineering of new genetic material in a safe manner by $\gamma$-retroviral or lentiviral vectors or nonviral methods using electroporation or transposon/transposase systems; adequate clinical grade expansion of genetically modified T cells in presence of cytokines, like IL-2, IL-7, IL-12 and/or IL-15; unhindered trafficking of transferred $\mathrm{T}$ cells to the tumor; and long-term survival of transferred $\mathrm{T}$ cells within the host.

Tisagenlecleucel, CD19-targeting CAR T cells, was the first to receive the US FDA approval in 2017 for the treatment of children and young adults with leukemia. The CAR T cell therapy has proven to be the most successful in treating blood tumors due to CD19-expressing B cell leukemias and lymphomas. The CD19-specific CAR T cell therapy has also shown promise in patients with refractory chronic lymphocytic leukemia, where CAR-modified CD19-specific T cells expanded to a level of more than 1000-times higher than the initial engraftment level in vivo. Additionally, findings in adolescents and adults with relapsed acute lymphoblastic leukemia showed effectiveness of CD19-targeted therapy in maintaining remission [13]. Studies have also shown that this type of adoptive CAR $\mathrm{T}$ cell therapy is beneficial in patients with relapsed or chemotherapy refractory B-cell leukemias [13]. Although hematologic malignancies make up the majority of successful attempts at CAR T cell therapy, some of the most vital lessons were learned from the study of solid tumors during early clinical trials. The overall challenge of using CAR T cell therapy on solid tumors is that there are few clear CAR target antigens. There are additional challenges for transferred CAR $\mathrm{T}$ cells that include environmental oxidative stress, nutritional depletion, acidic $\mathrm{pH}$ and hypoxia as well as immunosuppressive soluble factors, cytokines and immune cells (suppressive regulatory $\mathrm{T}$ cells, tumor-associated macrophages or myeloid-derived suppressor cells). Presently, there are several ongoing clinical trials focusing on CAR T cells for the treatment of solid tumors, including ovarian cancer, pancreatic cancer and mesothelioma. The presence of neoantigens on the surface of solid tumors signify thrilling new targets for CAR therapy in that their expression is restricted to tumor cells. One example is that of the epidermal growth factor receptor variant III (EGFRvIII) identified as a neoepitope expressed on glioblastomas [14]. To achieve user-defined diverse $\mathrm{T}$ cell responses, attempts are also underway to develop a universal programmable inducible CAR system that simultaneously encompasses the ability to switch targets, without the need for re-engineering the $\mathrm{T}$ cells, with a capacity to fine-tune $\mathrm{T}$ cell activation in response to multiple antigens [15]. 


\section{Limitations of CAR T cell therapy}

The CAR T cell therapy has its challenges in managing damage to normal tissues due to release of $\mathrm{T}$ cell peripheral tolerance, and severe systemic cytokine-release syndrome (CRS) leading to $T$ cell overactivation [13]. The CRS has been observed specifically with CD19-targeted CAR T cells, CARs targeting B cell maturation antigen, and CD22-specific CAR T cells. Symptoms of CRS range from flu-like to severe life-threatening indications caused by an overinflammatory response. More mild CRS symptoms include fever, fatigue, headache, rash, arthralgia and myalgia [13]. Another toxicity associated with CAR T cell therapy is tumor lysis syndrome, which appears during the peak of $T$ cell activity, leading to hyperuricemia, hyperkalemia, hyperphosphatemia and hypocalcemia. In addition, mild to life-threatening neurologic complication of cerebral edema has also been listed across various clinical studies focusing on CD19- and B cell maturation antigen-specific CAR T cells, even though some neurologic toxicities related to CD19-specific CAR T cells are characterized as reversible [13]. These complexities with intrinsic safety issues curtail the efficacy of existing $\mathrm{T}$ cell-based therapies. These issues may also jeopardize the outcomes of the recently proposed personalized mutanome vaccines aiming to elicit strong antitumor $\mathrm{T}$ cell responses [16]. Moreover, despite therapeutic improvements offered by genetically engineered antitumor $\mathrm{T}$ cells from a cancer patient, bolstered by immune checkpoint blockade antibodies, the 5-year survival rate after metastasis remains low in most malignancies $(<25 \%)$ other than leukemia and desmoplastic melanoma [8]. The CAR T cell therapy also presents a major limitation in that an exclusive CAR T cell product need to be produced for each patient, which is labor intensive and too personalized for widespread clinical use. Patients with rapidly developing advanced stage tumors may not afford to wait for the generation of individualized CAR T cells. It is clinically not favorable to use commercially available allogeneic $\mathrm{T}$ cells as they run the risk of causing graft-versus-host disease (GVHD).

\section{Evolution of CAR natural killer cell therapy}

To overcome the GVHD issue or cytokine storm associated with T cell-based treatments, the use of natural killer (NK) cells, which are nonspecific but highly cytotoxic immune effectors, is being explored. The NK cells also have the ability to maintain their antitumor functions outside of CAR targets, unlike CAR T cells, which decreases the risk of relapse following acquisition of resistance driven by the loss of CAR-targeted antigens. This aspect opens the door for the use of CAR NK cells as strong immunotherapeutic anticancer agents. Indeed, in published NK cell adoptive transfer clinical trials to date, the majority have not shown GVHD induction after infusion. Thus, NK cells and their CARs are actively being researched for their potential roles in tumor therapy. The NK cells use multiple effector mechanisms of action including cytokine-mediated toxicity via IFN- $\gamma$ and TNF- $\alpha$, granule-mediated cytotoxicity via granzymes and perforin, and antibody-dependent cellular cytotoxicity mediated by CD16. They also appear to represent on a per cell basis the most efficient effector cytolytic lymphocytes against tumors [17]. A range of NK-cell based applications have been explored including adoptive NK cell transfer, checkpoint blockade, combination therapies using monoclonal antibodies, bispecific antibodies that recognize NK cell receptors and tumor, gene transfer of transgenic cytokines and genetically engineered NK cells to express recombinant CARs. Research into CAR NK therapy has lately taken off as it provides potential for specificity and manipulation with the CAR-targeted tumor antigens along with other antitumor activities exhibited by their native receptors. With the use of NK cells, human leukocyte antigen matching is less of a concern than with T cells. Therefore, one donor/NK cell line could be developed to provide a cure to multiple patients without the issues of allogenic transplantation. Another advantage with the use of CAR NK is that NK cells have a limited mature lifespan much shorter than T cells. This ideally could limit any long-term adverse recognition effects, while maintaining antitumor activity. The US FDA-approved several investigational new drug applications for CAR NK cells are being explored. One such example is FATE-NK100, which employs genetically modified NK cells expressing the maturation marker CD57 and the activating receptor NKG2C. A challenge with implementing CAR NK and other NK cell applications stem from a lack of robust understanding of how NK cells reach their full antitumor cytolytic potential by overcoming the initial immune tolerance of its inhibitory receptors and triggering of its activating receptors. Once present in the tumor microenvironment, NK cells still need support in the form of cytokines to proliferate, to remain activated and to overcome immunosuppressive cytokines secreted by the tumor. Thus, before NK cells can become a prominent player in cancer immunotherapy, much work needs to be done to address questions concerning their source, recruitment, activation and costimulation. 


\section{Prospects of combination immunotherapy}

In the context of tumor-relevant models of unmutated self-antigens, tumor-infiltrating $\mathrm{CD} 8^{+} \mathrm{T}$ cells were found to provide essential 'help' to quiescent intratumoral NK cells by eliciting their antitumor function that eradicate antigen-escape tumor variants [5]. The NK cells in the proximity of activated $\mathrm{CD}^{+} \mathrm{T}$ cells showed functional remodeling exhibited by upregulation of effector, tissue-migratory and signaling molecules. This work suggests that tapping innate and adaptive lymphocyte teamwork in conjunction with lymphocyte-stimulatory pharmacological strategies, such as bortezomib [18] or engineered Notch ligand multivalent clusters [19], would be key to successful immunotherapy.

We are in an exciting time for the cancer immunotherapy field. The last two decades saw tremendous progress in our understanding of lymphocyte biology and in translating this to clinical trials. The results are eagerly awaited from numerous clinical trials that are under way to see whether improved responses can be attained by combination therapy approaches. The biggest challenges we face now are in targeting tumor heterogeneity displayed in terms of their tissue and immune landscape [20], understanding the microbiotal and psychosocial dynamics of immune cells, and properly managing the clinical safety issues. With the pace the field is progressing, one can anticipate that in near future we will have optimized immunotherapeutic regimens tapping the tremendous antitumor capacities of CAR T and CAR NK cells, bolstered by their intrinsic functional cooperativity and immune checkpoint blockade strategies. Such tailored combination immunotherapy regimens supported by the latest technological advancements in immunogenomics, cancer mutanome and immune engineering should deliver the expected outcomes of durable clinical responses. The prospective regimens would have the potential to fulfill the hope of an effective cancer therapy that could elicit long-term remissions where patients exhibit no signs of cancer for the remainder of their life span.

Financial \& competing interests' disclosure

This work was supported by funds to A Shanker by the following NIH grants (grant numbers U54 CA163069, U54 MD007593, SC 1 CA182843 and R01 CA175370). AN Renrick and ZT Dunbar are supported by NIH training grants (grant numbers T32 HL007737 and R25 GM059994, respectively). The authors have no other relevant affiliations or financial involvement with any organization or entity with a financial interest in or financial conflict with the subject matter or materials discussed in the manuscript apart from those disclosed.

No writing assistance was utilized in the production of this manuscript.

\section{References}

1. Ehrlich P. Ueber den jetzigen stand der Karzinomforschung. Ned. Tijdschr. Geneeskd. 5, 273-290 (1909).

2. Uyttenhove C, Maryanski J, Boon T. Escape of mouse mastocytoma P815 after nearly complete rejection is due to antigen-loss variants rather than immunosuppression. J. Exp. Med. 157(3), 1040-1052 (1983).

3. Schreiber R D, Old L J, Smyth M J. Cancer immunoediting: integrating immunity's roles in cancer suppression and promotion. Science 331(6024), 1565-1570 (2011).

4. Guerra N, Tan Y X, Joncker N T et al. NKG2D-deficient mice are defective in tumor surveillance in models of spontaneous malignancy. Immunity 28(4), 571-580 (2008).

5. Shanker A, Verdeil G, Buferne M et al. CD8 T cell help for innate antitumor immunity. J. Immunol. 179(10), 6651-6662 (2007).

6. Topalian S L, Rosenberg S A. Therapy of cancer using the adoptive transfer of activated killer cells and interleukin-2. Acta Haematol. 78(Suppl 1), 75-76 (1987).

7. Leach D R, Krummel M F, Allison J P. Enhancement of antitumor immunity by CTLA-4 blockade. Science 271(5256), 1734-1736 (1996).

8. Ribas A, Wolchok J D. Cancer immunotherapy using checkpoint blockade. Science 359(6382), 1350-1355 (2018).

9. Snyder A, Makarov V, Merghoub T et al. Genetic basis for clinical response to CTLA-4 blockade in melanoma. N. Engl. J. Med. 371(23), 2189-2199 (2014).

10. Freeman G J, Long A J, Iwai Y et al. Engagement of the PD-1 immunoinhibitory receptor by a novel B7 family member leads to negative regulation of lymphocyte activation. J. Exp. Med. 192(7), 1027-1034 (2000).

11. Luksza M, Riaz N, Makarov V et al. A neoantigen fitness model predicts tumour response to checkpoint blockade immunotherapy. Nature 551(7681), 517-520 (2017).

12. Shin D S, Zaretsky J M, Escuin-Ordinas H et al. Primary resistance to PD-1 blockade mediated by JAK1/2 mutations. Cancer Discov. 7(2), 188-201 (2017). 
13. June C H, O’Connor R S, Kawalekar O U, Ghassemi S, Milone M C. CAR T cell immunotherapy for human cancer. Science 359(6382), 1361-1365 (2018).

14. Newick K, Moon E, Albelda S M. Chimeric antigen receptor T-cell therapy for solid tumors. Mol. Ther. Oncolytics 3, 16006 (2016).

15. Cho J H, Collins J J, Wong W W. Universal chimeric antigen receptors for multiplexed and logical control of $\mathrm{T}$ cell responses. Cell 173(6), 1426-1438.e1411 (2018).

16. Sahin U, Türeci O. Personalized vaccines for cancer immunotherapy. Science 359(6382), 1355-1360 (2018).

17. Mehta RS, Rezvani K. Chimeric antigen receptor expressing natural killer cells for the immunotherapy of cancer. Front. Immunol. 9 , 283 (2018).

18. Shanker A, Pellom ST Jr., Dudimah DF et al. Bortezomib improves adoptive T-cell therapy by sensitizing cancer cells to FasL cytotoxicity. Cancer Res. 75(24), 5260-5272 (2015).

19. Biktasova A K, Dudimah D F, Uzhachenko R V et al. Multivalent forms of the Notch ligand DLL-1 enhance antitumor T-cell immunity in lung cancer and improve efficacy of EGFR-targeted therapy. Cancer Res. 75(22), 4728-4741 (2015).

20. Thorsson V, Gibbs DL, Brown SD et al. The immune landscape of cancer. Immunity 48(4), 812-830.e814 (2018). 